\title{
DEVELOPMENTS IN MAKING SPACE ACCESS RAPID AND AFFORDABLE USING A PLASMA RAILGUN*
}

\author{
D. Wetz ${ }^{\xi}$, I. McNab, F. Stefani, D. Motes, and J. Parker \\ Institute for Advanced Technology \\ The University of Texas at Austin \\ 3925 W. Braker Ln., Suite 400 \\ Austin, TX 78759
}

\begin{abstract}
For the last four years, the Institute for Advanced Technology has been working on the development of a plasma driven electromagnetic launcher (EML), for economic access to space [1]. The research is focused on overcoming setbacks experienced in the early developmental days of plasma-driven EMLs, which prevented researchers from obtaining muzzle velocities in excess of $6 \mathrm{~km} / \mathrm{s}$ [2]. The possibility of achieving muzzle velocities in excess of $7 \mathrm{~km} / \mathrm{s}$ with an EML make its use attractive and cost-efficient means for launching small $(\sim 10 \mathrm{~kg})$ microsatellites into low Earth orbit. For that reason, the research being performed is funded as part of a multidisciplinary university research initiative (MURI) by the United States Air Force Office of Scientific Research (AFOSR). In the summer of 2007, a muzzle velocity of $5.2 \mathrm{~km} / \mathrm{s}$ was achieved with no evidence of restrike arcs or bore ablation, the effects of which are believed to limit the velocity of plasma railguns to no more than $6 \mathrm{~km} / \mathrm{s}$. Since then, a series of modifications have been made to the railgun bore to improve its performance and lifetime. Some of those modifications, and the experimental results obtained as a result, are discussed here.
\end{abstract}

\section{INTRODUCTION}

In the early days of electromagnetic launch (EML) research, experimentalists were under the assumption that they could use plasma-armature railguns to accelerate payloads up to muzzle velocities in excess of $10 \mathrm{~km} / \mathrm{s}$. During the initial studies, velocities of only $4-5 \mathrm{~km} / \mathrm{s}$ were demonstrated for medium-bore $(25-50 \mathrm{~mm})$ railguns operating at typical accelerations of $400-600 \mathrm{kG}$ [3],[4], and only $6-7 \mathrm{~km} / \mathrm{s}$ velocities were obtained in smaller bore guns with accelerations of $1 \mathrm{MG}$ or greater [5][6]. It was unclear for many years why velocities in excess of $5 \mathrm{~km} / \mathrm{s}$, at modest accelerations, could not be achieved until the 1980s, at which point several causes for the velocity ceilings were discovered and understood.

The velocity ceiling for plasma armature railguns was found to be a direct consequence of ablation of the bore insulators, which causes the bore to fill up with a hot, dense, neutral gas [7]. This gas does not affect the performance of the railgun until, at high velocities, the voltage across the railgun breech increases to the point where conditions for high-voltage breakdown are met. When this occurs, additional plasma armatures, known as restrike or secondary arcs, are formed well behind the main armature. Ideally, the restrike arcs are not pushing against anything and should rapidly merge with the main armature, which is pushing the payload. However, in practice, the secondary armatures are retarded by viscous drag as they push the ablation products in the bore. This drag prevents the restrike arcs from catching up to the main armature - causing current, and thereby acceleration force, to be lost in the restrike arc - preventing any future acceleration of the payload.

Several suggestions for overcoming the bore ablation problem were made [7] and implemented [8]. However, as soon as positive effects were starting to be observed, funding shifted to solid-armature tactical railguns, and plasma-armature railgun programs were discontinued. The Institute for Advanced Technology (IAT) at the University of Texas at Austin has resumed the research into plasma-driven railguns as part of a multidisciplinary university research initiative (MURI) sponsored the United States Air Force Office of Scientific Research (AFOSR). For its part, the IAT has set up a proof-ofprinciple experiment aimed at launching $5 \mathrm{~g}$ polycarbonate projectiles to muzzle velocities in excess of $7 \mathrm{~km} / \mathrm{s}$ using a $7 \mathrm{~m}$ long laboratory-style electric launcher. The IAT's technique for eliminating bore ablation involves a three-pronged approach that includes using magnetic augmentation to reduce power dissipation in the plasma, using high-purity alumina insulators to raise the ablation parameter of the bore, and using preacceleration to prevent ablation of the bore materials at low velocity [1]. Figure 1 contains plots of the ablation parameter, calculated using equation (1), as a function of time for a simple and augmented railgun. It is shown that without the use of augmentation as well as pre-injection, it is impossible to eliminate insulator ablation, even when insulators with a high ablation threshold like alumina are used.

\footnotetext{
${ }^{*}$ Work supported by the U.S. Air Force Office of Scientific Research (AFOSR) under contract number DOA-8910.

६ email: david_wetz@iat.utexas.edu
} 


$$
f=\frac{V_{\text {armature }} I_{\text {armature }} t i m e^{0.5}}{\text { 4length } h_{\text {plasma }} \text { height } t_{\text {plasma }}}
$$
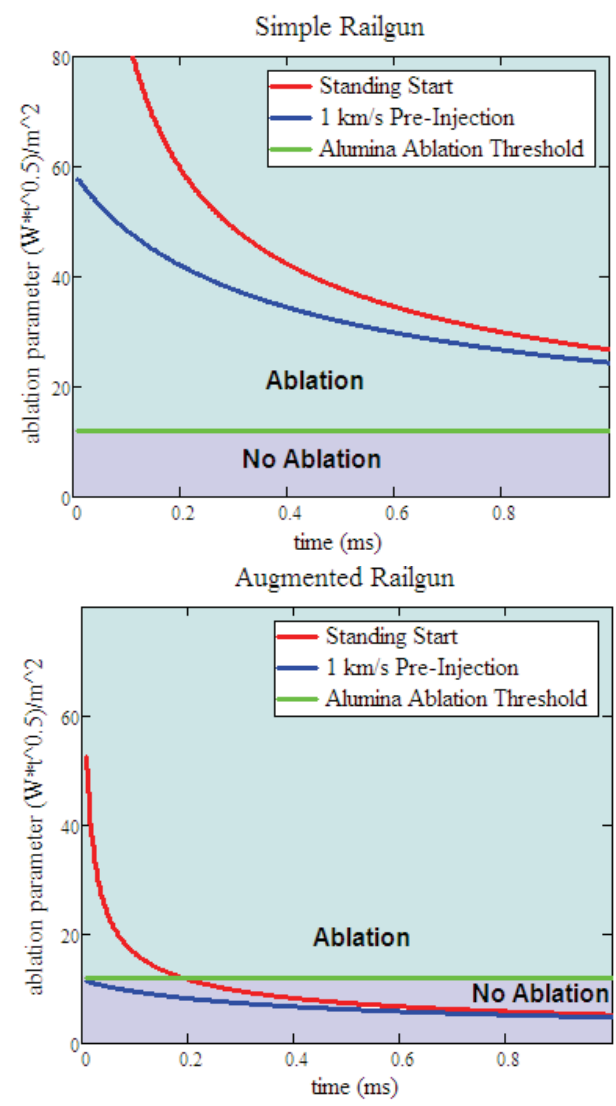

Figure 1. Ablation threshold as a function of time for a simple railgun (top) and an augmented railgun (bottom).

In addition to the efforts by the IAT, Texas Tech University is researching a distributed power supply configuration, which improves EML efficiency and further suppresses restrike arc phenomena when longer EMLs are used [9], and the Universities of Minnesota and New Orleans are investigating ways to overcome the aerothermal loads associated with launching payloads to such muzzle velocities. The results presented in this paper are limited to those obtained by the IAT.

\section{EXPERIMENTAL SETUP}

In the summer of 2007, a series of four experiments were performed in which muzzle velocities in excess of $5.2 \mathrm{~km} / \mathrm{s}$ were achieved with no evidence of bore ablation or restrike arcs. Figures 2 and 3 contain a solid model and a photograph of the experimental setup at the IAT. Figure 4 shows a cross section of the railgun core that was set up in the IAT's medium-caliber launcher (MCL) at that time. Details regarding the pre-injector power supply, pre-injector, augmentation crossover, and inner rail breech are given in [1] and will not be discussed here.

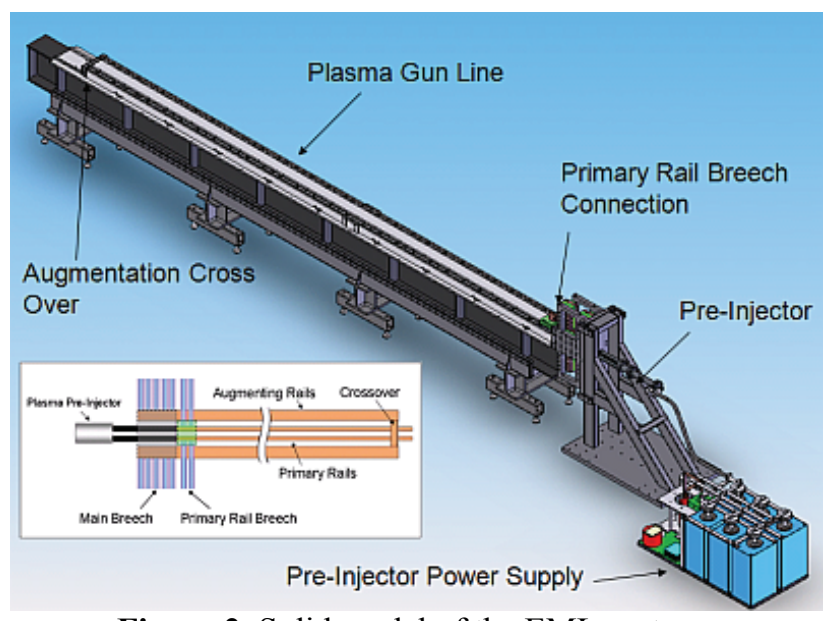

Figure 2. Solid model of the EML system.

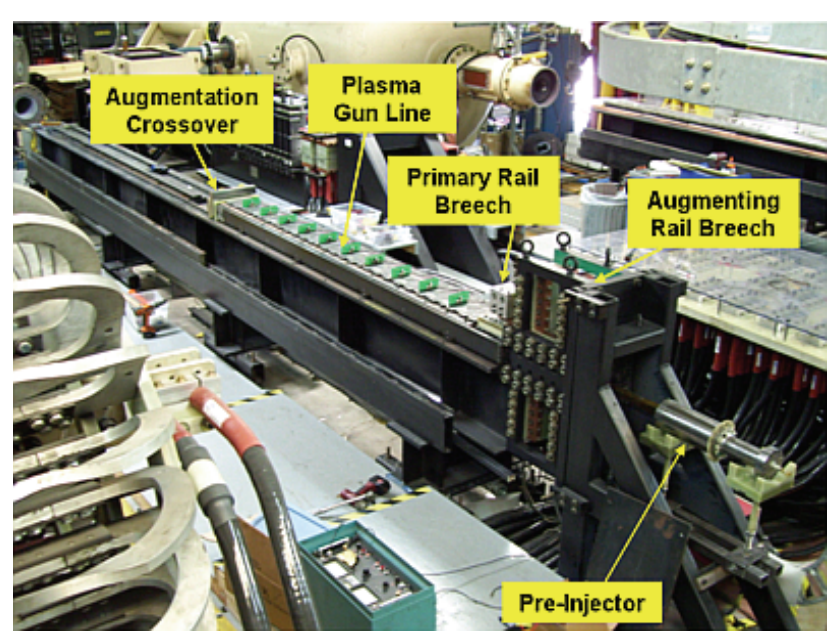

Figure 3. Constructed system in a $3 \mathrm{~m}$ long configuration.

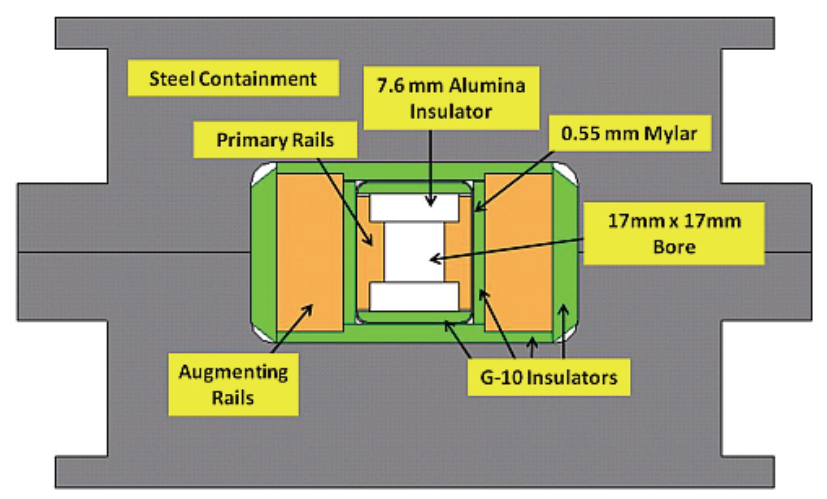

Figure 4. Plasma gun core.

In all the IAT's experiments, the MCL has been converted from a one-turn railgun into a two-turn, independently augmented railgun. The core tested in 2007 consisted of a set of large outer augmenting rails that enclose an inner core consisting of primary rails, alumina insulators, and polyolefin heat shrink. The latter 
provides some electrical isolation, but more importantly the ability to evacuate the bore to roughly 25-40 torr.

Initially, the inner core insulators were $99.5 \%$ pure alumina $\left(\mathrm{Al}_{2} \mathrm{O}_{3}\right)$ tiles that were $7.6 \mathrm{~mm}$ thick, $15.2 \mathrm{~cm}$ long, and stacked axially down the length of the gun. This grade of alumina has a measured ablation resistance of greater than $12 \mathrm{MWs} \mathrm{M}^{0.5} / \mathrm{m}^{2}$. Despite the excellent electrical results [1] obtained from the launcher, at the conclusion of each of the initial experiments, all the alumina insulators inside the inner core were catastrophically damaged, as shown in Figure 5.

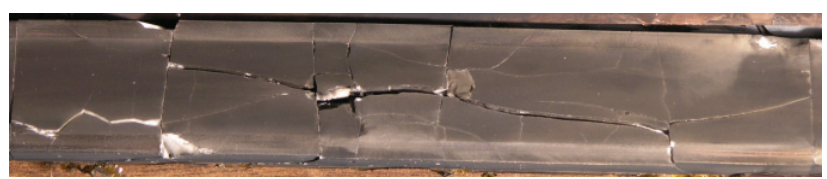

Figure 5. Broken alumina tile.

It was known prior to construction that a significant preload on the ceramic tiles would be required to prevent breakage; however, the required amount could not be applied with the initial design for two reasons. The first and dominant reason concerns the MCL's steel containment structure. The structure was designed to contain railgun bores intended for solid-armature research, which means the containment was designed to apply a significant preload in the horizontal direction but not in the vertical direction, since the forces in solidarmature EMLs are horizontal, as opposed to those in plasma-armature EMLs, which are isotropic inside the bore. What that means is that even with enough preload in the horizontal direction, the initial design did not provide adequate preload in the vertical direction, allowing the tiles to break when subjected to the large plasma pressure, estimated to be around $15 \mathrm{ksi}$. The second problem is with regard to the polyolefin heat shrink. It was mentioned that horizontal preload is required with solid-armature EMLs, but the amount of core interference, applied by driving in oversized side G10 insulators, required to obtain suitable preload is very small, on the order of $0.15 \mathrm{~mm}$, and can be applied by hand using a hammer. In this design, due to the thickness and sponginess of the heat shrink, roughly $0.75 \mathrm{~mm}$ of interference had to be applied, which was too much to apply by hand.

Though the goal of this research is to achieve velocities in excess of $7 \mathrm{~km} / \mathrm{s}$ and not to work on bore lifetime issues, it became a requirement due to financial limitations, which make it impossible to perform a significant number of experiments if a gun's worth of tiles must be procured for each shot. The problem could easily be fixed with a new containment design that is optimized to apply uniform preloads, but again the budget did not allow for it. With that in mind, modifications were made to the existing core and assembly procedure to improve the survivability of the bore components. The redesigned core is shown in Figure 6.

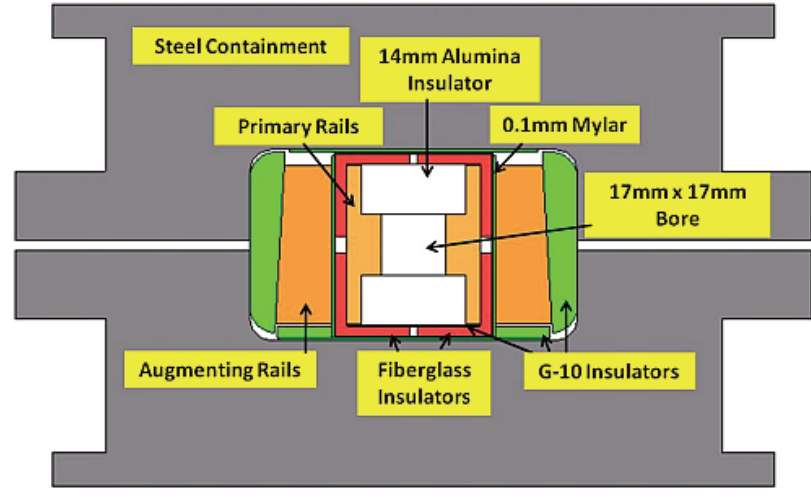

Figure 6. Redesigned plasma gun core.

The changes to the core include increasing the thickness of the ceramic insulators to $14 \mathrm{~mm}$, increasing the height of the step on the primary rails to accommodate the increased tile thickness, replacing the thick heat shrink with a thinner Mylar overwrap, adding a taper to the outer edge of the augmenting rails, and adding a mirrored taper to the G10 side insulators. The additional thickness of the ceramic tiles increases their stiffness by a factor of roughly seven. Additionally, a slight chamfer was added to the tile in the corner where it sits on the rail and the length of each tile was reduced to $50 \mathrm{~mm}$. The addition of the taper to the augmenting rails and the side insulators increases the mechanical advantage so that a sufficient preload to the ceramic tiles can be applied, and since a thinner and stiffer overwrap is being used, the required interference tolerance has been reduced. The last change involves the placement of fiberglass corner guards around each corner of the core. These prevent any plasma that leaks out of the bore from burning through the Mylar overwrap and causing breakdowns from the primary rails to the augmenting rails or containment structure.

Additionally, a hydraulic press fixture, shown in Figure 7, was designed to mount onto the gun and drive in the side insulators and upper containment halves with a greater deal of accuracy and consistency then was previously applied by simply hammering them in by hand.

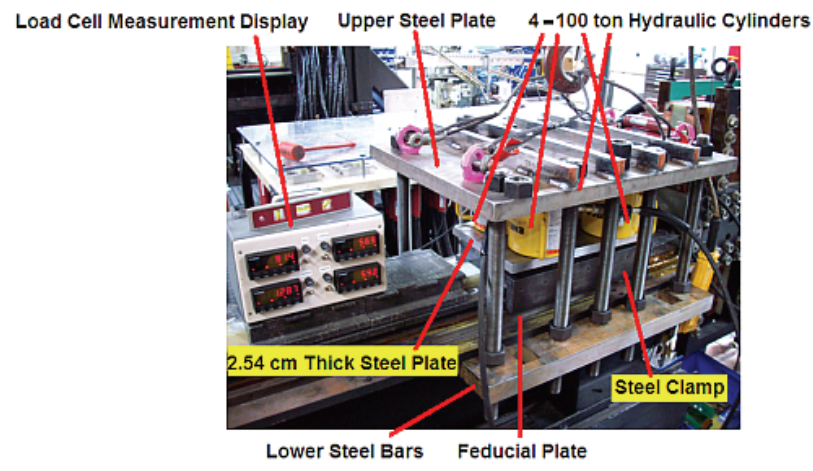

Figure 7. Hydraulic press fixture. 
The fixture mounts underneath the feducial plate on which the MCL containments are mounted and this reacts the load which is applied downward on both the insulators and the containment. A two step process is used to assemble the gun. First, each of the side insulators are driven into place on a single pass down the gun. This applies a significant preload to the tiles in the horizontal direction. A second pass is then made to push on the upper steel containments and compress the tiles in the vertical direction. Pressurex ${ }^{\circledR}$ pressure film was put into the core to measure the amount of compression in both the horizontal and vertical directions. The image of the film shown in Figure 8, indicates that roughly $15 \mathrm{ksi}$ is applied rather uniformly across the tiles in both directions. Though it is not being shown here, the similar use of Pressurex ${ }^{\circledR}$ film on the assembly of the original core design revealed good compression of the lower tiles but almost a complete lack of compression on the upper tiles.

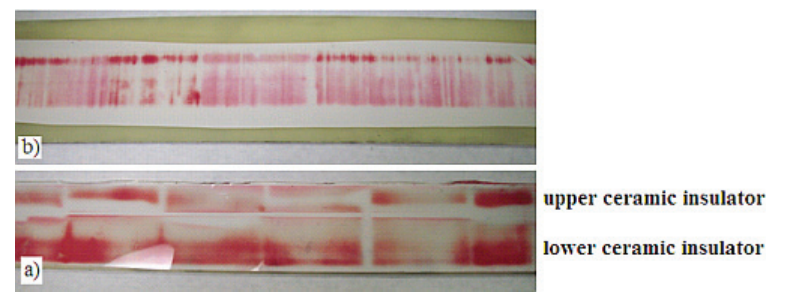

Figure 8. High Pressurex ${ }^{\circledR}$ film between the a) upper G10 insulator and steel containment and b) primary and augmenting rails.

\section{EXPERIMENTAL RESULTS}

Once the core had been redesigned such that the ceramic tiles were being properly preloaded, a build of the core was performed and a shot was taken in a $7 \mathrm{~m}$ configuration. Field enhancements were installed roughly $4 \mathrm{~cm}$ from the primary rail breech to ensure that the plasma armature was created ahead of the primary rail breech but not near the muzzle as has been a problem in the past due to the small bore dimensions. The field enhancements, before and after the shot, are shown in Figure 9. The first $5.3 \mathrm{~m}$ of the build contained ceramic insulators while the remainder of the gun contained G10 fiberglass-epoxy insulators. After the test, roughly $85 \%$ of the ceramic tiles were perfectly intact. The remainder had either minor or severe cracking. A photograph of the unfolded bore is shown in Figure 10.
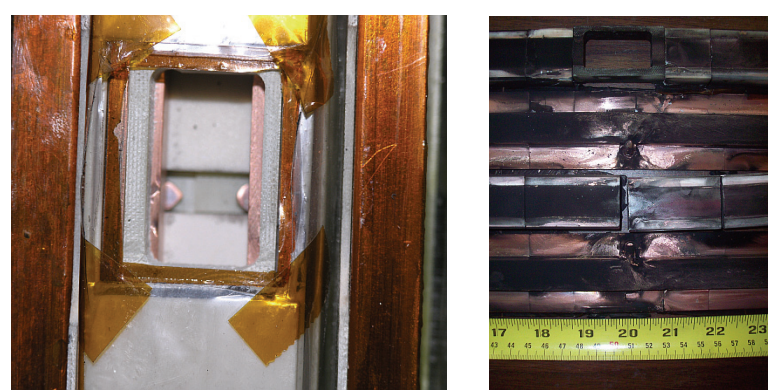

Figure 9. Field enhancements in the bore.

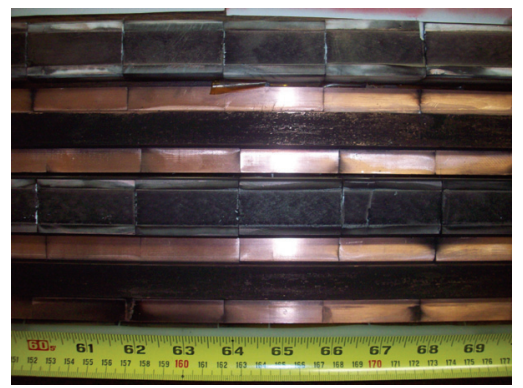

Figure 10. Photograph of the bore unfolded after the shot.

The current traces from the shot are shown in Figure 11 and the B-dot measurements are shown in Figure 12. Figure 13 shows a plot of the velocity and position of the armature vs. time. The B-dot traces display no evidence of restrike arcs, but integration of the traces reveals that despite the addition of field enhancements near the breech, a second arc was formed at the muzzle as soon as the primary current was discharged into the rails. Since the rail spacing is so small (17 mm), it takes less than $3 \mathrm{kV}$ to break down the rails at pressures up to 40 torr. It had been hoped that the addition of the field enhancements would restrict the breakdown to only their location eliminate dwell there, but neither was found to be the case. The erosion of the field enhancements indicates that, in addition to the main armature (which traveled down the bore) and the arcs (which formed at the muzzle), a stationary arc more than likely dwelled at the field enhancements, robbing current from the main armature. The structure of all the measurements recorded with B-dot probes beyond B-dot 10 reveals evidence of multiple armature formations in that location, making it impossible to discern velocity information from them. Between B-dots 10 and 11 correlates with the transition from ceramic to G10 insulators. The last measured velocity was an average velocity of $3.66 \mathrm{~km} / \mathrm{s}$ between B-dots 9 and 10 . 


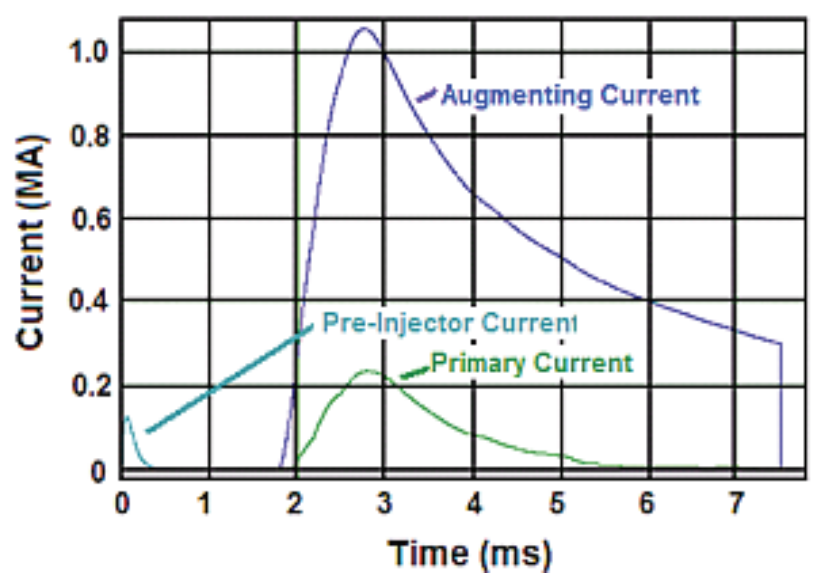

Figure 11. Current waveforms.

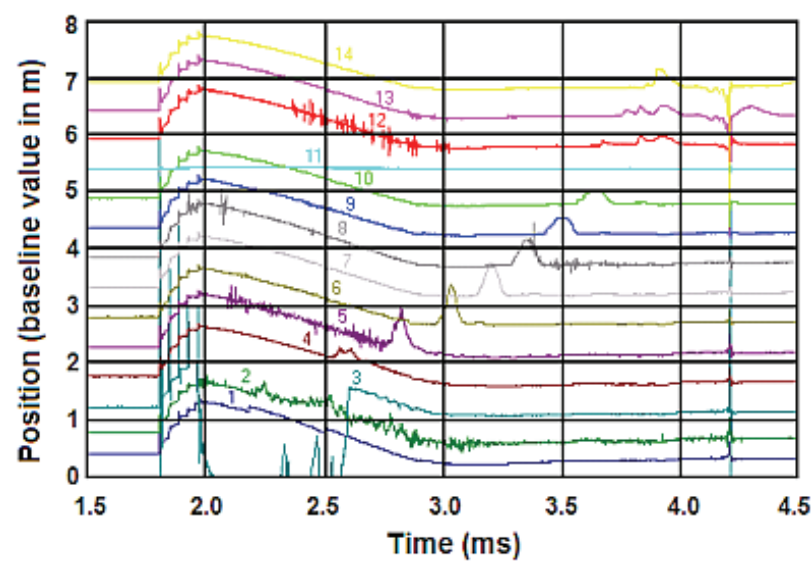

Figure 12. B-dot measurements in a waterfall plot, where the baseline value of the plot is the B-dots' position in $\mathrm{m}$.

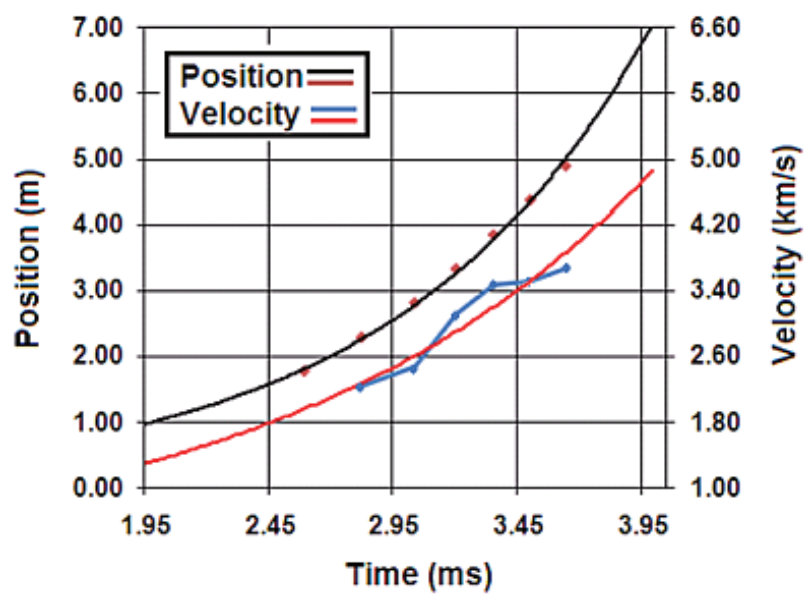

Figure 13. Plot of armature velocity and position vs. time as extrapolated from the B-dots.

Despite the measured velocity of $3.66 \mathrm{~km} / \mathrm{s}$, there was no evidence of a hypervelocity impact through the $3 \mathrm{~mm}$ witness plate at the muzzle, and the only explanation is that the polycarbonate projectile broke up in the bore, more than likely at the location of a welded joint in the primary rails at roughly $3 \mathrm{~m}$.

\section{SUMMARY}

The recent tests have shown that the modifications made to the MCL core design have improved the lifetime of the ceramic insulators considerably. The addition of field enhancements near the breech was not found to eliminate the formation of stationary arcs near at the muzzle of the gun. In future tests, full-length rails without a joint will be used, and a new fuse design will be implemented to reduce the voltage at the muzzle.

\section{ACKNOWLEDGMENT}

This material is based upon work supported by the AFOSR under award number DOA-8910. Any opinions, findings, and conclusions or recommendations expressed in this publication are those of the authors and do not necessarily reflect the views of the AFOSR.

\section{REFERENCES}

[1] D. Wetz, F. Stefani, J. Parker, and I. McNab "Advancements in the Development of a PlasmaDriven Electromagnetic Launcher," 14th International Electromagnetic Launch Symposium (EML), Victoria, BC, Canada, June 10-13, 2008.

[2] J. V. Parker, "Why plasma armature railguns don't work (and what can be done about it)," IEEE Trans. Mag., vol. 25, no. 1, pp. 418-424, 1989.

[3] K. A. Jamison and D. M. Littrell, "Performance characteristics of a high velocity, $25 \mathrm{~mm}$ railgun," IEEE Trans. Mag., vol. 31, no. 1, pp. 168-173, 1995.

[4] J. J. Scanlon et al., "Analysis of experimental data form a $50-\mathrm{mm}$ railgun driven by a 5-MJ capacitor power supply," IEEE Trans. Mag., vol. 29, no. 1, pp. 859-864, 1993.

[5] N. Kawashima et al., "Stable and reproducible production of high velocity projectile in ISAS railgun [HVPAC], "IEEE Trans. Mag., vol. 29, no. 1, pp. 431-434, 1993.

[6] E. Drobyshevski et al. "Experiment on simple railgun with the compacted plasma armature," IEEE Trans. Mag., vol. 31, no. 1, pp. 295-298, 1995.

[7] J. V. Parker, "Why plasma armature railguns don't work (and what can be done about it)," IEEE Trans. Mag., vol. 25, no. 1, pp. 418-424, 1989.

[8] M. Schulman et al., "HART hypervelocity augmented railgun test facility," IEEE Trans. Mag., vol. 29, no. 1, pp. 505-510, 1993.

[9] R.W. Karhi, J.J. Mankowski, and M. Kristiansen, "Analysis of Distributed Energy Railguns to Suppress Secondary Arc Formation," 14th International Electromagnetic Launch Symposium (EML), Victoria, BC, Canada, June 10-13, 2008. 